Lexis Vol. XLV (1) 2021: 263-285

\title{
Cuerpo y afecto en Noches de adrenalina (1981) de Carmen Ollé
}

\author{
Camilo Rubén Fernández-Cozman \\ https://orcid.org/0000-0002-7474-8666 \\ Universidad de Lima \\ crferna@ulima.edu.pe \\ Katherine Geraldine Medina Rondón \\ https://orcid.org/0000-0002-1826-2354 \\ Universidad Nacional de San Agustin de Arequipa \\ kmedinar@unsa.edu.pe \\ Nardy Luz Rosado Lazo \\ https://orcid.org/0000-0002-3247-1902 \\ Universidad Nacional de San Agustín de Arequipa \\ nrosado@unsa.edu.pe
}

\section{Resumen}

Este artículo plantea un análisis de Noches de adrenalina de la poeta peruana Carmen Ollé, a partir de la noción de cuerpo performático y afecto. El sujeto poético cuestiona los roles impuestos a las mujeres por la sociedad androcéntrica y manifiesta el desplazamiento migratorio de la periferia (Lima) a Europa (el centro). Asimismo, Ollé propone la liberación del cuerpo apoyada en teóricos de la Anti-Psiquiatría y la Psiquiatría 
Radical como una crítica implícita a la Psiquiatría, el Psicoanálisis y la religión. Por ello, postula la emergencia de un deseo queer.

Palabras clave: cuerpo performático, migración, feminismo, deseo queer

Body and affect in Nights of Adrenaline (1981) by Carmen Ollé

\begin{abstract}
This article presents an analysis of Nights of Adrenaline by the Peruvian poet Carmen Ollé based on the notion of performatic body and affect. The poetic subject questions the imposed roles to women by androcentric society and manifests the migratory shift from the periphery (Lima) to Europe (the center). Likewise, Ollé proposes body liberation based on Anti-Psychiatry and Radical Psychiatry theorists as an implicit criticism to Psychiatry, Psychoanalysis and religion. For this reason, she postulates the emergence of a queer desire.
\end{abstract}

Keywords: Performatic body, migration, feminism, queer desire

Carmen Ollé nació en Lima en 1947 y es una de las escritoras peruanas más notables del siglo XX. Aunque formó parte del parricida movimiento Hora Zero, su poesía no se enmarca en la estética del poema integral, que fue teorizada por Juan Ramírez Ruiz (uno de los fundadores del mencionado grupo poético) como una amalgama del todo individual con el todo universal (Ramírez Ruiz 1971: 110). Además, en este tipo de poema, preponderan la oralidad del lenguaje callejero y el rompimiento de los géneros literarios. Por eso, en un mismo texto conviven por igual lo lírico, lo narrativo y lo ensayístico. Dicha forma de poetizar solo guarda semejanza con la escritura de Ollé en el ámbito de la reflexión sobre el proceso migratorio (en este caso, de Lima hacia Europa). Recordemos que en los poemarios iniciales de Hora Zero es importante la experiencia de la migración desde las provincias hacia Lima (por ejemplo, En los extramuros del mundo de Enrique Verástegui o Un par de vueltas por la realidad de Juan Ramírez Ruiz). En cambio, la raigambre erótica de los textos de Carmen Ollé sí guarda relación con el libro 
Monte de goce de Verástegui, miembro ilustre de Hora Zero, quien escribe esta obra alejado de la teoría de la representación, manejada por sus adláteres horazerianos. Conviene aclarar, en ese sentido, que Noches de adrenalina propone otros recorridos para el erotismo: la función disruptora del cuerpo de la mujer dentro de lo heteronormativo, una crítica a los roles impuestos a las mujeres por la sociedad patriarcal, la presencia de un deseo queer y la actuación de un cuerpo performático antes que una presencia sensible. Esa propuesta se desarrolla en un contexto de liberación de los cuerpos que se ubica como heredero ya lejano de mayo del 68. En el Perú, la mal llamada poesía erótica ${ }^{1}$, debe entenderse como un momento especial, donde la escritura de las mujeres cobra visibilidad. Ollé y otras autoras (como María Emilia Cornejo, Patricia Alba, Rocío SilvaSantisteban, Mariela Dreyfus, Dalmacia Ruiz Rosas y muchas más) son las voces más notorias de los años setenta y ochenta del siglo pasado. Ollé ha publicado poemarios, novelas, cuentos y también se ha desempeñado como profesora universitaria, tallerista de escritura creativa y activista por los derechos de la mujer. Noches de adrenalina, poemario publicado en 1981, se instala como una reflexión periférica de una mujer instalada en París, la ciudad luz, como representante de la racionalidad, la alta filosofía y las bellas artes. En contraste, la misma ciudad es también aquella donde se mueven los migrantes latinoamericanos; en esa ciudad textual existe una filosofía fisiológica y escatológica de la mano de Bataille, Artaud y Sade o donde los poemas están atravesados por una dicción expresionista y colindante con un informe médico: "Los discursos científicos son «escabrosos», contienen y ponen en escena de manera condensada las luchas por los significados y las prácticas" (Haraway 1995: 349), es decir, en dichos ámbitos institucionalizados, se instrumentalizan

\footnotetext{
1 El cuerpo fue el eje central de la poesía femenina, dado que se encontraba inscrito en la condición de desigualdad, de los mitos sobre la diferencia de clases y el deseo sexual. En este sentido, se ha leído erróneamente como "poesía erótica” a la poesía que aborda el cuerpo, reduciendo su discurso a la supuesta facticidad del cuerpo. Esta ignora la carga social-político-existencial subyacente que permite un quiebre epistemológico capaz de cuestionar ideales sociales.
} 
las experiencias de la enfermedad, la mortalidad y lo viviente. En ese mismo sentido, la noción de “sexo”, para Foucault (2007), está relacionada con las disciplinas del cuerpo y con la regulación de las poblaciones. A su vez, la sexualidad es concebida como un dispositivo. "Sexo" y "sexualidad" se articulan como un micropoder sobre el cuerpo, e incluso como regulaciones ejercidas sobre poblaciones enteras. En este operativo no se trata acerca de la amenaza de muerte como en el poder soberano, sino de la administración de la vida. En el caso de Noches de adrenalina consiste en cómo el lenguaje médico ejerce una biopolítica sobre los cuerpos y las poblaciones: “en la unión del «cuerpo» y la «población», el sexo se convirtió en blanco central para un poder organizado alrededor de la administración de la vida y no de la amenaza de muerte" (Foucault 2007: 178). En este sentido, proponemos que, en el mencionado poemario, el cuerpo adquiere una condición médica, performativa de resistencia y cambio que deviene en una nueva forma de ser mujer.

\section{Medicalización de la vida}

Foucault (1996) propone que las sociedades modernas son "Estados médicos abiertos” (80), es decir, que son espacios donde se ejerce la medicalización de la vida. No se trata ya solo de responder a las demandas del enfermo o la existencia de enfermedades, sino que son imposiciones al individuo que van más allá del carácter médico e implican modificaciones y características del aire, el agua, la vivienda y los alimentos. En ese sentido: "la medicina se impone al individuo, enfermo o no, como acto de autoridad” (Foucault 1996: 75). Por ejemplo, exámenes médicos para conseguir un nuevo empleo o el acusado de un crimen debe someterse necesariamente al diagnóstico psiquiátrico. Lo que ocurre es que la medicina hace un movimiento desde lo clínico hacia lo social, hacia una práctica social: "El control de la sociedad sobre los individuos no se opera simplemente por la conciencia o por la ideología, sino que se ejerce en el cuerpo, con el cuerpo" (Foucault 1996: 87). Esto es importante porque en Noches de adrenalina hay descripciones clínicas de un cuerpo que 
entra en la madurez, pero esto nos indica que la medicalización de la vida, en cuanto a su aspecto social, incide en el control, persuasión y manipulación de los cuerpos. En ese tenor, también puede leerse la idea de moralidad e higiene del cuerpo, las condiciones de trabajo y vivienda, la alimentación y el ritmo de vida (Foucault 1996: 78).

Foucault (1979: 104-105) afirma que el autoerotismo es el lugar donde se produce una lucha entre los niños y los padres, entre los infantes y las instancias de control. Esto quiere decir que la masturbación es una forma de escapar a esa vigilancia y control. El poder que actúa reprimiendo la sublevación del cuerpo sexual hace en otras instancias un control-estimulación, es decir, que no siempre se trata de la punición. Por ejemplo, a través de la moda y los estándares de belleza, se posiciona la idea de que un cuerpo esbelto y bronceado es un cuerpo fuerte y sano, en otras palabras, deseable dentro de la sociedad capitalista como fuerza laboral. Ergo, convierte a los individuos en seres "capaces de trabajar, de asegurar la constancia de la fuerza laboral, su mejoramiento y reproducción” (Foucault 1996: 80). En Noches de adrenalina, la locutora personaje $\mathrm{e}^{2}$ opta por el autoconocimiento de su propio cuerpo como una forma de liberación: "étrangère! /mujer que atraviesa un verano desolado/y se acaricia el sexo como un espectro/ desnudo en una galería” (Ollé 2014: 64), pero ese mismo cuerpo es medicalizado e insertado dentro del circuito del capital en tanto cuerpo "subdesarrollado”, migrante (Lima-París), sucio e indeseable:

El cuerpo humano se vio doblemente englobado por el mercado: en primer lugar, en tanto que cuerpo asalariado, cuando el hombre vendía su fuerza de trabajo, y en segundo lugar por intermedio de la salud. Por consiguiente, el cuerpo humano entra de nuevo en un mercado económico, puesto que es susceptible a las enfermedades y a la salud, al bienestar o al malestar, a la alegría o al sufrimiento, en la medida que es objeto de sensaciones, deseos, etcétera (Foucault 1996: 81).

2 Fernández (2009) distingue entre locutor (a) personaje (que se evidencia cuando se utiliza la primera persona o la segunda a quien el yo pudiera dirigirse) y locutor (a) nopersonaje (que se manifiesta cuando se emplea la tercera persona). 
Los primeros versos de Noches de adrenalina han sido vinculados con una crítica al Psicoanálisis, pues hay una mención explícita: “¿Por qué el psicoanálisis olvida el problema de ser o no ser/ gorda/ pequeña/ imberbe/ velluda/ transparente/ raquítica/ ojerosa” (Ollé 2014: 7). Sin embrago, creemos que la crítica más fuerte que se hace en el poemario es a la Psiquiatría y no al Psicoanálisis. Sobre la base de las ideas de Foucault, podemos afirmar que el Psicoanálisis es una despsiquiatrización, "pero al mismo tiempo reconstitución del poder médico, productor de verdad” (Foucault 2007: 56). En todo caso, hay que fijarse en que "las relaciones de poder constituyen el a priori de la práctica psiquiátrica: condicionan el funcionamiento de la institución manicomial, distribuyen en su interior las relaciones entre los individuos, rigen las formas médicas de intervención" (Foucault 2007: 58). En suma, las vías por las que Noches de adrenalina propone la liberación de los cuerpos son la Anti-Psiquiatría ${ }^{3}$, la Psiquiatría Radical ${ }^{4}$ y el erotismo entendido como una forma de resistencia frente al capital: “Tener 30 años no cambia nada salvo aproximarse al ataque/ cardíaco o al vaciado uterino. / Dolencias al margen/ nuestros intestinos fluyen y cambian del ser a la nada" (Ollé 2014: 7). No solo se trata de que la mujer pueda nombrar las partes de su cuerpo de manera libertaria o del envejecimiento del cuerpo medicalizado, sino del deterioro del cuerpo asalariado

\footnotetext{
3 Szasz (1976, 1974, 1993), uno de los referentes de la Anti-Psiquiatría y pionero en descubrir la represión de locura, defiende la posición de que los denominados trastornos mentales no poseen una correspondencia física (corazón, hígado, etc.), anota además que al no ser la mente un órgano, no existe enfermedad y en correspondencia no debería existir hospitalización o cura. Al no existir enfermedad mental, nos encontraríamos con cambios meramente conductuales, existiendo, de esta manera, conductas "normales" y “anormales". Por consiguiente, los diagnósticos mentales son aplicados a personas cuya actuación conductual incomoda u ofende a una sociedad determinada, estigmatizando a la persona a la que se le ha otorgado esta etiqueta punitiva.

4 Entiéndase por Psiquiatría Radical una tendencia dentro de la Antipsiquiatría, influenciada mayoritariamente por la psicología humanista y menos por el psicoanálisis. Laing (1987) y Cooper (1967) afirman que la psiquiatría es una forma más de opresión social, similar a otras instituciones de la sociedad patriarcal tal como el capital, la familia, la escuela, etc. y que los diagnósticos mentales son arbitrarios en el sentido de que dependen de una determinada sociedad, ideología, momento histórico y formación profesional de turno.
} 
—entiéndase dentro de la economía feminista propuesta por Picchio (2005), donde el trabajo se construye desde una perspectiva neutra, recuperando los elementos femeninos invisibilizadas, particularmente el trabajo doméstico y de cuidados-. Esta clave es fundamental para entender por qué Noches de adrenalina está atravesada por lo escatológico. Por ejemplo, en estos versos el flujo de los intestinos (fisiología/escatología) hace una comparación implícita con el flujo de capitales (trabajo asalariado/usura).

Por otra parte, ¿qué quiere decir, para Foucault, el hecho de que exista resistencia? En las relaciones de poder debe producirse resistencia, pues esta es la que cambia las relaciones de poder. Si no, estamos dentro del campo de la obediencia. Dicha resistencia puede desempeñar "el papel de adversario, de blanco, de apoyo, de saliente para una aprehensión" (Foucault 2007: 116). Cuando Foucault habla de resistencia no solo se refiere a una contrapartida de la dominación, sino a puntos irregulares que "surca[n] las estratificaciones sociales y las unidades individuales" (2007: 117). Según Lemke (2011), para Foucault, se trata de que la resistencia provoca formas de subjetivación enmarcadas como: "social oppositions between men and women, conflicts on the definition of health and disease, reason and madness, in the rise of ecological movements, peace movements, and sexual minorities" (51).

En el caso de Noches de adrenalina, y siguiendo a Rosi Braidotti, se puede decir que se posiciona una nueva subjetividad femenina en torno a "cuestiones relativas a los derechos y a la autoridad, es decir, al poder. Se establece, pues, una conexión entre la política y la epistemología" (Braidotti 2004: 15). Braidotti también apunta que es determinante el sentido experiencial y afirma que el cuerpo "constituye el sitio de intersección de lo biológico, lo social y lo lingüístico" (2004: 16), puesto que el cuerpo es el lugar de localización, entendiendo también el cuerpo como una "entidad socializada, codificada culturalmente" (16) y no como una instancia esencialista. En ese sentido, se ha hecho la diferencia entre hombres y mujeres atendiendo a la oposición binaria y primaria de los sexos (varón/mujer), pero aquí también debe considerarse lo étnico, 
la clase social, la identidad de género, etc. En varios momentos de Noches de adrenalina se erige esta subjetividad femenina como categoría política, experiencial y teórica o, como afirma Nancy K. Miller, “sujeta al cambio" (Braidotti 2004: 14) En otras palabras, como afirma Teresa de Lauretis, se trata de un "sujeto feminista femenino" (citado en Braidotti, 2004: 14). Esta experiencia de las mujeres y la categoría "mujer" son deconstruidas en Noches de adrenalina, donde se propone una especie de emancipación que significa abandonar la culpa del dogma religioso y quebrar la cultura dominante: "Lo que brota de natural de un cuerpo aplastado/no se resume en fáciles categorías como divino o decadente/todo WC es un jardín oculto/oler a orín reconforta/el cristianismo lleva hoy el peso de estos olores/y muchos gramos de bicarbonato para las náuseas" (Ollé 2014: 17). Ollé propone algunas salidas para lograr la mencionada emancipación, pero es importante que señalemos el concepto "devenir mujer" como el proceso inicial para la desterritorialización del sujeto dominante:

El devenir mujer es subversivo en tanto que supone un esfuerzo activo hacia la transformación de los signos, las prácticas sociales y las historias encarnadas de la feminidad institucionalizada blanca. Una aproximación deleuziana demanda renunciar a la búsqueda de una identidad modelada conforme al sujeto molar/sedentario para, en cambio, activar múltiples devenires alejados de la identidad (Braidotti 2005: 139).

Recordemos que, para Deleuze, la segmentación dura o molar está compuesta por segmentos donde todo parece contable y previsto, tanto el principio y el final de un segmento, como el pasaje de uno a otro: primero la familia, luego la escuela, después el ejército, más tarde la fábrica y, finalmente, la jubilación. Se trata de una línea rígida, dura, estable, cuyos segmentos están bien delimitados: familia-profesión, trabajo-vacaciones. 


\section{Proceso migratorio en Noches de adrenalina}

Noches de adrenalina es el relato de una mujer latinoamericana migrante que se mueve entre dos espacios de enunciación: Europa (por ejemplo, París como la ciudad soñada por los artistas e intelectuales o Menorca como punto intermedio) y el Perú (léase: la ciudad capital, Lima). El sujeto migrante (Cornejo Polar 1996) expande su territorio y habla desde diversos espacios de enunciación donde se superponen el centro y la periferia.

En el primer texto de Noches de adrenalina se ejerce una crítica a la cultura patriarcal occidental, pero también se hace un primer movimiento de contraste o descolonización. Se establece como representantes de esa cultura letrada a Jean Genet frente a la novelista Albertine Sarrazin, o a los líderes estudiantiles de la década del 60, como Daniel Cohn Bendit, Rudi Dutschke y Ulrike Meinhof frente a las camaradas marxistas y "subdesarrolladas" Elsa, Margarita y Sira: "Del botín que es la cultura me pregunto por el destino/ ¿Por qué Genet y no Sarrazine 5 ? o Cohn Bendit / Dutschke / Ulrike/ y no las pequeñas militantes que iluminaban mis aburridas / clases en la U/ ELSA MARGARITA SIRA" (Ollé 2014: 7).

Habíamos apuntado antes que es necesario no solo tener en cuenta la diferencia de los sexos, sino también lo étnico, la clase social y la identidad de género. En este primer poema de Noches de adrenalina, se refiere a tres estudiantes universitarias marxistas: "Marx aromaba en sus carteras como retamas frescas/ qué bellas están ahora calladas y marchitas [...] De día interrumpían las clases de metafísica con rabia/ y aplaudíamos esos cabellos sudorosos y negros sobre/ la espalda" (Ollé 2014: 8). Lo interesante no es solo

\footnotetext{
5 Para el presente ensayo no hemos podido revisar todas las ediciones de Noches de adrenalina, pero queremos apuntar que en la edición de PEISA (Ollé 2014) así como en la coedición de Flora Tristán/Lluvia (2005) se escribe mal el apellido de la novelista francesa Albertine Sarrazin que en la cadena semántica debe ir al lado de Jean Genet, al haber tenido estos dos personajes vidas disolutas y al margen de las normas sociales. Esta errata lleva a confusión con la novela Sarrasine de Balzac, incluso Carlos Villacorta (2016: 175) llega a mencionar al escritor de La comedia humana como una de las referencias intertextuales de Noches de adrenalina.
} 
saber que las mujeres peruanas en la década del 70 y el 80 participan activamente de la vida política, sino que el aroma de sus carteras era de retamas frescas y el color de sus cabellos eran oscuros. Estas mujeres -Elsa, Margarita y Sira- son estudiantes de la universidad estatal y libres sexualmente; pues frecuentan hoteles pobres donde liberan sus cuerpos al placer. En ese sentido, esos cuerpos políticos y sexuales son más contundentes y corporizados que una clase de metafísica. Pero el segundo verso citado: "qué bellas están ahora calladas y marchitas”, se puede leer en dos sentidos: o ellas han sido oprimidas y asimiladas por las estructuras capitalistas y patriarcales (obreras/madres) o han fallecido en la lucha política. Las dos posibilidades postulan el desencanto.

Esos “cabellos sudorosos”, así como otros versos, instalan una dicotomía entre lo sucio y lo limpio en Noches de adrenalina. Santiváñez afirma que la expresión: "El que más se lava es el que más apesta [proviene] de una consigna de París, mayo 68 y también del Movimiento Hippie de San Francisco [...] La cita aludida es una crítica frontal a la supuesta asepsia y pulcritud del orden burgués" (2016: 164). Por contraste, lo limpio es asociado a trabajos precarios que hacen los migrantes en países del llamado primer mundo. En ese sentido, lo limpio se asocia con la explotación capitalista y burguesa: "Bataille es quizás su guía pues, como Bataille, ella resueltamente anula la jerarquía que privilegia la limpieza sobre la suciedad, lo puro sobre lo impuro" (Franco 2016: 115). La usura es, sin duda, sucia y reprobable; pero, para el poder hegemónico, son sucios los migrantes y sus deseos: "nada de entonces [la infancia] puede penetrarme en el miedo/ como esta ciudad en la usura" (Ollé 2014: 28). Esta metáfora se enfoca en términos de desigualdad de clases y de género; además, erige la idea de que en lo que respecta al deseo no hay acumulación posible y solo se trata del derroche. Un síntoma del desgaste del cuerpo, como ya se ha mencionado, es la pérdida de los dientes o un ovario. Por ello, en Noches de adrenalina, la salubridad y sanidad de ese cuerpo se pone en entredicho no como marca negativa, sino como rompimiento con los cánones tradicionales de belleza y pulcritud, pero también como envejeci- 
miento del cuerpo asalariado. En este sentido, entendamos, a su vez, que el trabajo materno empuja a la subalternidad, al someter a la mujer a la invisibilidad política de los procesos encarnados por la normatividad que las envuelve. La maternidad es un trabajo sin remuneración que somete al cuerpo. Además, la mujer, en una sociedad, hostil, neoliberal y machista, se ve obligada a disfrutar de dicha maternidad.

Ollé también realiza una comparación entre los estudiantes de Lima y los migrantes, pues todos ellos son seres precarios, que casi viven en la orfandad, en la suciedad: "estudiantes = habitaciones inmundas/ lavabos + amasijo de pelos \& residuos de grasa” (Ollé 2014: 8-9). Al mismo tiempo, los trabajos de las latinoamericanas en París hacen que no puedan conocer otra ciudad que no sea la de la servidumbre: “¿Qué son los Campos Elíseos o la Gioconda sino el ménage/ delegado a las jóvenes muchachas del Tercer Mundo?/ Lavar pisos/ refregar las estrellas” (Ollé 2014: 11). París no era una fiesta ni tampoco era el lugar de la alta cultura ni de los grandes palacios, museos y conciertos: "Desde los jardines de la U imaginaba París como un barrio/ cálido donde alcanzar el espíritu de los impresionistas" (Ollé 2014: 22). Europa y sus urbes modernas son los espacios donde las mujeres migrantes latinoamericanas tienen que realizar trabajos asalariados para sobrevivir. Entonces, Occidente no es la promesa de la felicidad y el triunfo artístico para los migrantes, pues "en Italia roban en las estaciones del tren/ violan en Montmartre/ asaltan en el barrio chino/ París cansa" (Ollé 2014: 29-30). Tal como lo había planteado Cornejo Polar (1996), solo le queda al sujeto migrante el descentramiento y frente a eso dos opciones: la adecuación y el anclaje. Adecuación en tanto se trata de otras ciudades donde los roles se rigen por el capital, y la discriminación lingüística y étnica "en una ciudad extranjera/ donde el yo se improvisa como/ el único personaje en medio del estado policial” (Ollé 2014: 60). Este yo, como el cuerpo que analizamos en el contexto de vigilancia y represión, tiende a ser performático y al devenir. El anclaje es la reafirmación de antiguas identidades, en este caso, latinoamericanos, estudiantes pobres, marcados 
por la culpa de la religión, barbados que escuchan jazz y fuman marihuana: "en la noche cené puerco dulce en restaurantes vietnamitas/ y era como volver a la calle Capón en Lima, la necesidad/ absurda de reencontrarnos siempre a millas de distancia/ con una vaga identidad" (Ollé 2014: 22). Este anclaje, como advertimos, está caracterizado como una "vaga identidad" y es melancólico, nostálgico. Para Ahmed (2019), quien nos presenta al "inmigrante melancólico" como una figura atravesada por el "racismo infeliz" y la "felicidad multicultural”, este migrante es una forma de "ubicar la herida en determinado lugar” (323) y cómo la toma de conciencia sobre el racismo puede ser "diagnosticada como una forma de melancolía” (323).

\section{Cuerpo performático en Noches de adrenalina}

La locutora personaje habla de la maternidad, de criar a una hija pequeña, lavar pañales y de estar casada; roles de madre y esposa que se les exige a las mujeres dentro de la sociedad patriarcal, reduciendo el cuerpo de la mujer a algo biológico, irracional e hipersensible. El hecho de dar a luz hace que el cuerpo envejezca (por ejemplo, una cicatriz producto de la cesárea): "Mi vagina se llena de hongos como consecuencia del primer parto" (Ollé 2014: 7). A su vez, pérdida de los dientes e incluso del ovario son síntomas del deterioro del cuerpo, pero no solo eso. Este paso del tiempo significa para la mujer de Noches de adrenalina el ingreso pleno a un estadio de liberación del cuerpo y, sobre todo, el despojarse de los tabúes sociales y religiosos: "Noches de adrenalina, al indagar por la identidad a través de la mirada hacia el pasado y del cuerpo oculto, descubre el momento de quiebre entre esta infancia mística y la adultez erótica de la que habla Bataille” (Villacorta 2016: 178). Por ello, se puede entender la automutilación del cuerpo, como experiencia límite dentro de lo erótico, como brote referido a lo sagrado, la prohibición y la transgresión de las que nos habla Bataille. En esta instancia, podemos afirmar que el cuerpo de Noches de adrenalina no es una entidad sensible, entendiendo que: "El punto de partida 
de la significación es más bien un sujeto que toma posición sobre el cuerpo. Un sujeto que se ubica a distancia de su ser corporal y que a la vez lo sufre $[\ldots]$ un sujeto [...] capaz de tomar distancia" (López Maguiña 2016: 153).

Creemos que el cuerpo en Noches de adrenalina es un cuerpo performático. Judith Butler, en el ensayo "Performative Acts and Gender Constitution: an Essay in Phenomenology and Feminist Theory", señaló, por primera vez, lo performático en relación con la identidad de género. Dicha identidad no viene programada biológica ni ontológicamente, sino que se construye a través de una repetición estilizada de actos que se relacionan con acciones corporales. Estos actos performáticos son los que van generando una identidad fluida: "One is not simply a body, but, in some very key sense, one does one's body" (Butler 1990: 272). Uno no es simplemente un cuerpo, por el contrario, uno hace su propio cuerpo. Este cuerpo performático de Noches de adrenalina, y aquí coincidimos con López Maguiña, tiene capacidad de distancia, pero también tiene observación afectiva y compulsión escatológica. Como profundizaremos más adelante, la clave de la lectura marxista se encuentra en los cuerpos sucios y rechazados de la clase trabajadora migrante; del mismo modo, abordaremos la crítica a la Psiquiatría y el Psicoanálisis mediada por la recurrencia a corrientes críticas y contrapuestas como la Anti-Psiquiatría y la Psiquiatría Radical. En ese sentido, nos parece pertinente la lectura de Hernández, quien propone que Ollé sitúa "en obra la noción de texto y escritura como una performance, donde el cuerpo de la voz es también el cuerpo de una mujer adulta mirándose a sí misma en el espejo psicoanalítico del superyó" (2016: 135). Debemos subrayar que el cuerpo de Noches de adrenalina busca la liberación y justamente la liberación de ese superyó, entendido como la cultura y las normas sociales impuestas, pues el cuerpo también está atravesado por el engaño, el tabú y la prohibición social: "[Para Butler] la realización de la identidad de género, o de cualquier otra, como proceso de corporización se lleva a cabo de forma análoga a la de una realización escénica teatral" (Fischer-Lichte 2011: 57), es decir, 
la identidad de género es una experiencia colectiva, performática; a pesar de que las acciones se repiten, nunca son las mismas, así como tampoco lo son las experiencias que generan.

Lo performático, en comparación, con el devenir puede entenderse como algo diferente a la transformación o metamorfosis. En suma, lo performático, al igual que el devenir, es no teleológico y no continuo. En otras palabras, evita las semejanzas, la identificación, la imitación; rechaza el binarismo, va adoptando, rotando y cambiando sus múltiples identidades. En tal sentido, la performatividad alude al "poder del discurso para producir efectos mediante la reiteración” (citado en Ahmed 2015: 149). Dicha idea de Butler es desarrollada por Ahmed, quien afirma que:

Por un lado, lo performativo se refiere al futuro; genera efectos en la constitución o materialización de aquello que "todavía no" es. Pero, por otro lado, la performatividad depende de la sedimentación del pasado; reitera lo que ya se ha dicho, y su poder y autoridad dependen de cómo evoca aquello cuya existencia ya ha producido (2015: 149).

\section{Cuerpo y afecto en Noches de adrenalina}

Para la teoría del afecto, no existe "un cuerpo". Lo que existe son cuerpos en plural, puesto que el afecto también pone en juego el movimiento de los cuerpos y su relación con los sonidos, las cosas y los ambientes. La teoría del afecto critica la idea de que la racionalidad solo se origina con el pensamiento, pues desde Descartes se cree que la mente piensa y el cuerpo actúa; además, hay que tener muy en cuenta la idea spinoziana de cómo un cuerpo afecta a los otros cuerpos y cómo ese impacto produce un residuo que afecta al cuerpo (Ahmed 2019: 426). Por ejemplo, el dolor no forma la superficie de los cuerpos, pero es lo que hace más presente al cuerpo: “A través de experiencias sensoriales como el dolor llegamos a sentir nuestra piel como una superficie corpórea [...], como algo que nos mantiene separados de los otros" (Ahmed 2015: 53). Pero aquello que nos separa, también nos conecta. En ese sentido, se puede pensar 
la piel como conector y separador virtual (un registro múltiple de sensaciones) e incluso entender el cuerpo como un lugar desde donde se lee el afecto, pero este cuerpo debe ser entendido, antes que como organismo, como un proceso donde emerge el afecto. Entonces, el afecto es preconsciente, ligado al cuerpo, es potencial y energético, va más allá de lo discursivo y el significado, y vincula al cuerpo con los procesos sociales.

Por ello, una lectura de Noches de adrenalina que considere el afecto como potencia y virtualidad y en tanto que se ligue el cuerpo erótico con el cuerpo social es pertinente, pues en la obra de Ollé se puede percibir que el erotismo no es simple liberación del cuerpo, sino que esa mostración escatológica de lo erótico va de la mano de una crítica política que socava los roles impuestos a las mujeres dentro de la heteronormatividad: "la suciedad llega a ser el capítulo de mi existencia/que resiste a la lucidez del adulto,/el momento en que al levantarme la falda/sobrevino el castigo el miedo a la soledad/ resbalar en el sueño de lo imaginado/embriagado por sus propios olores.” (Ollé 2014: 58). La configuración de un deseo andrógino y de un deseo lésbico apunta a ese tenor, es decir, la construcción de un feminismo queer, como se explicará más adelante. En este sentido, una lectura de la migración y sus estragos en el propio cuerpo y el cuerpo social es necesaria, como habíamos apuntado anteriormente, puesto que el afecto también podemos relacionarlo con un movimiento decolonial y deconstructivo.

En Noches de adrenalina, la mostración de los genitales se mezcla con enfermedades o sus síntomas (presión baja, temperatura alta, aceleración del pulso, aliento seco y ácido, inflamaciones hepáticas, embolia, distensión de los músculos bucales, alteración de la retina, hinchazón del vientre) y procesos afectivos (melancolía, nostalgia, abandono, risa). Por ello, es remarcable que el cuerpo en Noches de adrenalina no es solo un cuerpo erótico, sino, como ha apuntado Roland Forgues, se trata de un cuerpo descrito de forma clínica y opuesto al confesionalismo (citado en Rowe 2016: 80), aunque en algunos pasajes del libro sí se producen confesiones a través del recuerdo y la culpa en la infancia y la adolescencia: "des- 
pués de masturbarme quería llorar de miedo y de vergüenza" (Ollé 2014: 55). Sara Ahmed (2015) ha analizado de manera detenida las funciones del miedo y la vergüenza. Por ejemplo, menciona que la invocación a la imagen de la familia tradicional durante la guerra es una forma como "la heterosexualidad se vuelve el guion que liga lo familiar con lo global” (222). Esto quiere decir que la unión entre hombre y mujer se torna en la instancia que mantiene, metafóricamente, integrada a la nación, en tanto reproducción de vidas, cultura y valores, y en ese contexto se entiende "el deslizamiento en las narrativas racistas entre el miedo a los extraños e inmigrantes (xenofobia), el miedo a las personas queer (homofobia) y el miedo a la mezcla racial (así como a otras uniones ilegítimas)” (222).

En Noches de adrenalina, la función del miedo es mediada a través de la culpa religiosa. Para la locutora personaje, no es solo la masturbación lo que le provoca miedo y vergüenza, sino que también le suscita miedo la mantención de la familia nuclear, el proceso migratorio y el deseo queer reprimido hasta esos momentos. Pero este miedo cambiará en las páginas finales del libro como veremos posteriormente. Para finalizar esta parte del análisis, traeremos a colación el concepto “devenir mujer”, puesto que esta teoría elude el binarismo de masculino y femenino y propone posicionamientos cambiantes y múltiples que Braidotti llama sujetos nómades: "la referencia a la «mujer» en el proceso de «devenir mujer» no se refiere a las mujeres empíricas, sino, en realidad, a posiciones topológicas, a grados y niveles de intensidad, a estados afectivos. El devenir mujer es el marcador de un proceso general de transformación, ya que afirma fuerzas positivas y niveles de conciencia nómada, rizomática” (2005: 103).

\section{Liberación del cuerpo en Noches de adrenalina: deseo queer}

El acto sexual está teñido de culpa por una imposición de la moral judeocristiana: "El miedo se mezcla a la cópula como un regocijo" (Ollé 2014: 16). Luego, se cita a Claude Steiner, uno de los representantes de la Psiquiatría Radical: “Opresión + engaño = alineación// 
Opresión + conocimiento = cólera” (Ollé 2014: 16). Es decir, no solo se trata de la opresión, sino que aseveran que la opresión no existe, y que la vida está hecha de esa manera y que no hay otras formas de vivir; si uno se da cuenta de este procedimiento, cae en la cólera e indignación. Según Steiner, para poder liberarnos, necesitamos no solo tener conocimiento de la opresión, sino también contar con apoyo humano. En ese sentido, la culpa y el dolor vienen mediadas por la religión: “A los 15 años se está de pie ante una cruz un arquetipo/ del dolor/ me arrodillo beso la punta de esos pies sangrantes” (Ollé 2014: 17). Es provocadora la imagen de la muchacha adolescente, tal vez virginal, que besa los pies sangrantes en un movimiento de fetichización del cuerpo lacerado, crucificado y agonizante.

En otro poema se puede leer: "Fui masoquista/ a solas gozadora del llanto en el espejo del WC/ antes que La muerte de la Familia nos diera el alcance" (Ollé 2014: 8). Al respecto, es interesante anotar que el libro de David Cooper, al que se alude, se inscribe como uno de los máximos representantes de la Anti-Psiquiatría como corriente del pensamiento. En ese libro se erige una crítica a la estructura familiar burguesa y represiva, en cuanto, se construye sobre la base del patriarcado y la monogamia; es decir, la locutora personaje declara que el masoquismo le ocasionaba placer y a la vez culpa, pero que la lectura de Cooper le ayuda a proponer alternativas de liberación personal como el amor, la política de la comunidad o la locura.

$\mathrm{Al}$ acercarnos a las últimas páginas del poemario, Carmen Ollé (2014) cita un fragmento de Safo: "estuve enamorada de ti, Atis, una vez, hace/ ya tiempo, aunque me parecías una niña/ menudita ...” (48); la locutora personaje agrega “y no muy limpia” (48), como lo examinamos antes, lo sucio se asocia a los trabajadores migrantes precarios. Es concordante que el deseo lésbico se exprese aquí a través de la metáfora de lo no muy limpio, es decir, ese deseo lésbico está posicionado por la sociedad patriarcal dentro de las prácticas sucias, al igual que la masturbación, el sadomasoquismo, el fetichismo y el sexo anal: "no he vencido el fastidio en el per anus/ y no 
me resuelvo a hacerlo con su miembro obstruyendo/ el intestino" (Ollé 2014: 42). Así como se muestra un deseo lésbico, también hay un deseo andrógino, entendiendo que el deseo como el cuerpo es performático y nómade: "yo la sodomizo sin desgarramiento ni crueldad/ sin dolor/ sólo presiono su espalda/ mi pequeña almeja no da para más/ (tampoco la sobriedad) / andrógino en otro cielo otra piel” (Ollé 2014: 46). Entiéndase, además, al andrógino, a través del mito platónico aparecido en el Banquete (Platón 2010), como un ser completo que reunía en sí a los dos sexos.

Por consiguiente, creemos que Carmen Ollé no solo participa de la segunda oleada del feminismo con su celebrada máxima "lo personal es político”, sino que también se inscribe en la tercera oleada feminista que busca problematizar la figura de la mujer e instaurar una suerte de feminismo queer: "la palabra «queer» es performativa: ha adquirido significados nuevos a través de la repetición (Butler 1997c). «Queer», término que antes se usaba para insultar (cuando ser queer era ser no nosotros, no hetero, no normales, no humanos), se ha convertido en un nombre para una orientación política alternativa" (Ahmed 2015: 224).

El recorrido de un deseo queer que se postula en Noches de adrenalina, también se puede rastrear en la novela Las dos caras del deseo de Carmen Ollé (1994). Susana Reisz dice sobre esta obra que es "la primera novela (peruana) que aborda abiertamente una temática lesbiana” (Reisz 1996: 234). Ada, la protagonista de la novela, vive en una prisión. Lima y su ambiente puritano y prohibitivo; la oscilación del personaje frente a los diversos cuerpos del deseo, y su identidad construida bajo el concepto de la dominación, hacen que Ada se escinda entre lo que desea y su vida normal. En ese sentido, para Rocío Ferreira, el personaje de Ada se inscribe desde una “mirada deseante queer” (2016: 215).

Como se apuntó anteriormente, el concepto de sujeto nómade también se despliega en Noches de adrenalina. El final del libro es el que se muestra más expeditivo respecto a un deseo queer: "H.M. es cierto: como a una mujer veleidosa/ la amo/ y siento esa náusea de no saber amarla/ nuestro deseo es rígido y poco inflamable ante/ un 
cuerpo femenino/ la madurez ha obturado lo que en la adolescencia/ era transparente/ él/ ella” (Ollé 2014: 67). La pareja hombre (H.) y mujer (M.) que se ha desplegado a lo largo del poemario y que en un principio tenía la cualidad de lo cierto, de lo real ${ }^{6}$, en este final parece empezar a resquebrase; la locutora personaje reafirma la idea de que el deseo queer se asocia con lo sucio, lo repugnante, y eso le produce “esa náusea”. Los últimos versos son de total desencanto, la heteronormatividad ("él/ella”) asimilada en la infancia y la adolescencia, ahora en la adultez se ha queerizado (a la vez es un deseo lésbico y andrógino y, por ello, es performático, nómade y es un devenir), pero este deseo aún es nuevo: "rígido y poco inflamable”. Ahmed (2018) propone la vuelta a un feminismo lesbiano porque: "Hacerse lesbiana es queerizar a la mujer quitándosela al hombre. Crear un mundo para mujeres es dejar de ser mujeres para” (304). Y esas parecen ser las palabras finales de Noches de adrenalina.

\section{Referencias bibliográficas}

Ahmed, Sara

2015 La política cultural de las emociones. México: Universidad Nacional Autónoma de México.

Ahmed, Sara

2018 Vivir una vida feminista. Barcelona: Edicions Bellaterra.

Ahmed, Sara

2019 La promesa de la felicidad. Una crítica cultural al imperativo de la alegría. Buenos Aires: Caja negra.

BRAIDOTTI, Rosi

2004 Feminismo, diferencia sexual y subjetividad nómade. Barcelona: Gedisa.

\footnotetext{
6 Aunque H.M. también pueden ser las siglas de una escritora o un escritor o algún personaje del mundo real efectivo que no hemos podido descifrar. Mariela Dreyfus apunta que se trataría de Henry Miller "el ambiguo H.M. del último poema” (Dreyfus 2016: 46).
} 
BRAIDOTTI, Rosi

2005 Metamorfosis. Hacia una teoría materialista del devenir. Madrid: Akal.

BuTLER, Judith

1990 "Performative Acts and Gender Constitution: An Essay in Phenomenology and Feminist Theory". En Performing Feminisms: Feminist Critical Theory and Theatre. Ed., Sue-Ellen Case. Baltimore y London: The John Hopkins University Press. 270-282.

Cooper, David

1967 Psiquiatría y antipsiquiatría: Buenos Aires: Locus Hypocampus.

Cornejo Polar, Antonio

1996 "Una heterogeneidad no dialéctica: sujeto y discursos migrantes en el Perú”. Revista Iberoamericana. LXII. 176$177,837-844$.

Dreyfus, Mariela

2016 "No hay acto político inocente: hipermoralidad y mal en Noches de adrenalina, de Carmen Ollé". En Esta mística de relatar cosas sucias. Ensayos en torno a la obra de Carmen Ollé. Eds., Mariela Dreyfus, Bethsabé Huamán Andía y Rocío Silva-Santisteban. Lima: Latinoamericana Editores y Centro de Estudios Antonio Cornejo Polar, 43-51.

FERREIRA, Rocío

2016 "Subjetividades nómadas y queer durante la violencia política en tres novelas de Carmen Ollé, Aída Balta y Pilar Dughi”. En Esta mística de relatar cosas sucias. Ensayos en torno a la obra de Carmen Ollé. Eds., Mariela Dreyfus, Bethsabé Huamán Andía y Rocío Silva-Santisteban. Lima: Latinoamericana Editores y Centro de Estudios Antonio Cornejo Polar, 215-228.

FisCHER-Lichte, Erika

2011 Estética de la performativo. Madrid: Abada Editores.

Foucault, Michel

1979 Microfísica del poder. Madrid: Las Ediciones de la Piqueta.

Foucault, Michel

1996 La vida de los hombres infames. La Plata: Editorial Altamira. 
Foucault, Michel

2007 Historia de la sexualidad. 1-la voluntad de saber. México: Siglo Veintiuno.

FrANCO, Jean

2016 "Introducción a Noches de adrenalina". En Esta mística de relatar cosas sucias. Ensayos en torno a la obra de Carmen Ollé. Eds., Mariela Dreyfus, Bethsabé Huamán Andía y Rocío Silva-Santisteban. Lima: Latinoamericana Editores y Centro de Estudios Antonio Cornejo Polar, 111-115.

Haraway, Donna J.

1995 Ciencia, cyborgs y mujeres. La reinvención de la naturaleza. Madrid: Cátedra.

HERNÁNDEZ, Biviana

2016 "Noches de adrenalina, abyección y narcisismo". En Esta mística de relatar cosas sucias. Ensayos en torno a la obra de Carmen Ollé. Eds., Mariela Dreyfus, Bethsabé Huamán Andía y Rocío Silva-Santisteban. Lima: Latinoamericana Editores y Centro de Estudios Antonio Cornejo Polar, 117-137.

LAING, Ronald

1987 Razón, demencia y locura. Barcelona: Crítica

Lemke, Thomas

2011 Biopolitics. An Advanced Introduction. New York y London: New York University Press.

López Maguiña, Santiago

2016 "Noches de adrenalina de Carmen Ollé: cuerpo femenino y migración”. En Esta mística de relatar cosas sucias. Ensayos en torno a la obra de Carmen Ollé. Eds., Mariela Dreyfus, Bethsabé Huamán Andía y Rocío Silva-Santisteban. Lima: Latinoamericana Editores y Centro de Estudios Antonio Cornejo Polar, 151-160.

Ollé, Carmen

$1994 \quad$ Las dos caras del deseo. Lima: PEISA.

Ollé, Carmen

$2014 \quad$ Noches de adrenalina. 4ta. edición. Lima: PEISA. 
Picchio, Antonella

2005 "La economía política y la investigación sobre las condiciones de vida". En Por una economía sobre la vida. Aportaciones desde un enfoque feminista. Comps., G. Cairói Céspedes y M. Mayordomo. Barcelona: Icaria., 17-34.

Platón

2010 El banquete. Barcelona: Icaria Editorial.

RAMírez Ruiz, Juan

1971 Un par de vueltas por la realidad. Lima: Ediciones del movimiento Hora Zero.

Reisz, Susana

2016 “'Transgresión o negociación?: sexualidad y homoerotismo en la narrativa peruana reciente". En Esta mística de relatar cosas sucias. Ensayos en torno a la obra de Carmen Ollé. Eds., Mariela Dreyfus, Bethsabé Huamán Andía y Rocío SilvaSantisteban. Lima: Latinoamericana Editores y Centro de Estudios Antonio Cornejo Polar, 233-244.

Rowe, William

2016 "Carmen Ollé: mirar y oír". En Esta mística de relatar cosas sucias. Ensayos en torno a la obra de Carmen Ollé. Eds., Mariela Dreyfus, Bethsabé Huamán Andía y Rocío SilvaSantisteban. Lima: Latinoamericana Editores y Centro de Estudios Antonio Cornejo Polar, 79-103.

SANTIVÁÑEZ, Roger

2016 "Eros \& política: una lectura de Noches de adrenalina de Carmen Ollé". En Esta mística de relatar cosas sucias. Ensayos en torno a la obra de Carmen Ollé. Eds., Mariela Dreyfus, Bethsabé Huamán Andía y Rocío Silva-Santisteban. Lima: Latinoamericana Editores y Centro de Estudios Antonio Cornejo Polar, 61-173.

SzASZ, Thomas

1974 La fabricación de la locura. Barcelona: Kairos.

SzAsz, Thomas

1976 El mito de la enfermedad mental. Buenos Aires: Amorrortu. 
SzASz, Thomas

$1993 \quad$ Nuestro derecho a las drogas: en defensa de un mercado libre. Barcelona: Editorial Anagrama.

Villacorta, Carlos

2016 "Erotismo y espacio en Noches de adrenalina de Carmen Ollé: una lectura de Bataille y Bachelard”. En Esta mística de relatar cosas sucias. Ensayos en torno a la obra de Carmen Ollé. Eds., Mariela Dreyfus, Bethsabé Huamán Andía y Rocío Silva-Santisteban. Lima: Latinoamericana Editores y Centro de Estudios Antonio Cornejo Polar, 175-187.

Recepción: 27/07/2020

Aceptación: 04/12/2020 